\title{
Boundedness in a parabolic-elliptic chemotaxis system with nonlinear diffusion and sensitivity and logistic source
}

\author{
Giuseppe Viglialoro ${ }^{10}$ | Thomas E. Woolley ${ }^{2}$
}

${ }^{1}$ Dipartimento di Matematica e Informatica, Università di Cagliari, Italy

${ }^{2}$ Cardiff School of Mathematics, Cardiff University, Senghennydd Road, Cardiff, Cardiff CF24 4AG United Kingdom

\section{Correspondence}

Giuseppe Viglialoro, Dipartimento di Matematica e Informatica, Università di Cagliari, V. le Merello 92, 09123 Cagliari (Italy).

Email: giuseppe.viglialoro@unica.it

Communicated by: R. Bravo de la Parra

\section{Funding information}

Smart Cities and Communities and Social Innovation-ILEARNTV, Grant/Award Number: SCN_00307

MSC Classification: 35A01; 35B40; 35K55; 35Q92; 35K59; 92C17
In this paper, we study the zero-flux chemotaxis-system

$$
\left\{\begin{array}{lr}
u_{t}=\nabla \cdot\left((u+1)^{m-1} \nabla u-u(u+1)^{\alpha-1} \chi(v) \nabla v\right)+k u-\mu u^{2} & x \in \Omega, t>0, \\
0=\Delta v-v+u & x \in \Omega, t>0,
\end{array}\right.
$$

where $\Omega$ is a bounded and smooth domain of $\mathbb{R}^{n}, n \geq 1$, and where $m \in \mathbb{R}$, $k, \mu>0$ and $\alpha \leq 1$. For any $v \geq 0$, the chemotactic sensitivity function is assumed to behave as the prototype $\chi(v)=\chi_{0} /(1+a v)^{2}$, with $a \geq 0$ and $\chi_{0}>0$. We prove that for any nonnegative and sufficiently regular initial data $u(x, 0)$, the corresponding initial-boundary value problem admits a unique global bounded classical solution if $\alpha<1$; indeed, for $\alpha=1$, the same conclusion is obtained provided $\mu$ is large enough. Finally, we illustrate the range of dynamics present within the chemotaxis system in 1,2 , and 3 dimensions by means of numerical simulations.

\section{KEYWORDS}

asymptotic behaviour, boundedness, chemotaxis, global existence, logistic source, nonlinear parabolic systems

\section{1 | INTRODUCTION AND MOTIVATIONS}

This paper is dedicated to the following problem

$$
\begin{cases}u_{t}=\nabla \cdot\left((u+1)^{m-1} \nabla u-u(u+1)^{\alpha-1} \chi(v) \nabla v\right)+k u-\mu u^{2} & x \in \Omega, t>0, \\ 0=\Delta v-v+u & x \in \Omega, t>0, \\ \frac{\partial u}{\partial v}=\frac{\partial v}{\partial v}=0 & x \in \partial \Omega, t>0, \\ u(x, 0)=u_{0}(x) \geq 0 & x \in \Omega,\end{cases}
$$

where for the unknown $(u, v)=(u(x, t), v(x, t))$, the component $x$ belongs to a bounded and smooth domain $\Omega$ of $\mathbb{R}^{n}$, with $n \geq 1, t \geq 0$ and where $m \in \mathbb{R}$ and $k, \mu>0$. The function $u_{0}(x)=u(x, 0)$ is the initial value of $u$ and it is taken from $W^{1, \infty}(\Omega)$, while $\partial / \partial \nu$ indicates the outward normal derivative on $\partial \Omega$. Moreover, we assume that $\alpha \leq 1$ and that the function $\chi$ generalises the standard chemotactic sensitivity

$$
\chi(v)=\frac{\chi_{0}}{(1+a v)^{2}} \quad \text { with some } \quad \chi_{0}>0 \quad \text { and } \quad a \geq 0 .
$$

The mathematical formulation of this problem describes phenomena tied to chemotaxis models, which indicate the movement of a cell population at a point $x$ of an environment, identified as $\Omega$, and at an instant $t$ (ie, $u=u(x, t)$ ) when 
stimulated by a chemical signal (ie, $v=v(x, t)$ ), which is also distributed in the space and produced by the cells. In addition, the zero-flux boundary conditions on both $u$ and $v$ (the homogeneous Neumann boundary conditions) express that the interaction takes places in a totally insulated domain. ${ }^{1}$

Problem (1) is one of several generalisations of the following landmark model proposed by Keller and Segel in 1970 (see Keller and Segel ${ }^{2}$ and the more recent reviews $\left.{ }^{3,4}\right)$,

$$
\begin{cases}u_{t}=\Delta u-\chi \nabla(u \cdot \nabla v) & x \in \Omega, t>0, \\ \tau v_{t}=\Delta v-v+u & x \in \Omega, t>0, \\ \frac{\partial u}{\partial v}=\frac{\partial v}{\partial v}=0 & x \in \partial \Omega, t>0, \\ u(x, 0)=u_{0}(x) \geq 0 \text { and } v(x, 0)=v_{0}(x) \geq 0 & x \in \Omega,\end{cases}
$$

where $\chi>0, \tau \geq 0, \Omega \subset \mathbb{R}^{n}$, with $n \geq 1$, is a bounded domain with smooth boundary, and $u_{0}(x)=u(x, 0)$ and $v_{0}(x)=v(x, 0)$ are the initial cell distributions and chemical concentration, respectively. This system and many of its variants have been widely discussed by many authors, in terms of both the parabolic-elliptic (ie, $\tau=0$ ) and the parabolic-parabolic case (ie, $\tau=1$ ). All of these models may eventually lead to an uncontrolled gathering of cells in some localised spatial location; such phenomenon, known as chemotactic collapse, mathematically implies that $u$ becomes unbounded in one or more points of its domain, in finite time (blow-up time). In the parabolic-parabolic case, it is known that solutions to (2), in one spatial dimension, are global and uniformly bounded in time (see Osaki and Yagi ${ }^{5}$ ), while in the $n$-dimensional context, with $n \geq 2$, unbounded solutions have been detected in Horstmann and Wang ${ }^{6}$ and Winkler. ${ }^{7}$ Estimates for the blow-up time of such unbounded solutions are explicitly derived in Payne and Song. ${ }^{8}$

Further, if $\tau=0$ in the second equation of system (2) then Jäger and Luckhaus ${ }^{9}$ and Nagai $^{10}$ have shown that, under suitable assumptions, bacteria concentrations blow up in finite time. This blow up occurs in both radial and nonradial two-dimensional solutions.

Furthermore, it is worth mentioning that for the fully parabolic case important results concerning the existence of both bounded or unbounded solutions to chemotaxis-systems have also been attained when the first equation of system (2) is replaced by $u_{t}=\nabla \cdot(\phi(u) \nabla u)-\nabla \cdot(\psi(u) \nabla v)$. The occurrence of such different scenarios are studied in terms of the asymptotic behaviours of the ratio between the diffusion $\phi(u)$ and the sensitivity $\psi(u)$, as well as the space dimension. We refer, for instance, to Cieťslak and Morales-Rodrigo ${ }^{11}$ and Ciesťlak and Winkler ${ }^{12}$ for the parabolic-elliptic case and to previous studies $^{13-15}$ for the parabolic-parabolic one. Finally, for $\phi(u) \simeq u^{-m}$ and $\psi(u) \simeq u^{\alpha}$, with $m \geq 0$ and $\alpha \in \mathbb{R}$ and the second equation given by $0=\Delta v-M+u, M$ being the spatial mean of $u$, it is known (see Winkler and Djie ${ }^{16}$ ) that all the solutions of the corresponding zero-flux initial boundary problem are globally bounded if $m+\alpha<2 / n$, whereas if $m+\alpha>2 / n$ and $\Omega$ is a ball then unbounded solutions can be detected; in particular the value $2 / n$ represents the critical exponent for the quantity $m+\alpha$.

In order to remove Dirac delta-type singularities, the introduction of absorptive sources seems totally natural. The presence of the term $k u-\mu u^{2}$ in our problem (1) arises from the well known model of population dynamics (originally formulated by Pierre-François Verhulst $i^{17}$ ) concerning the self-limiting growth of a biological population. We remark that another commonly considered choice of the source, involving further zeros and replacing $k u-\mu u^{2}$, is given by the bistable expression $u(B-u)(u-A)$, with $0<A<B$; the corresponding Keller-Segel-type systems are investigated, for instance, in Funaki et $\mathrm{al}^{18}$ and Funaki and Tsujikawa, ${ }^{19}$ and shock-type movements or travelling fronts are detected (see also Marras and Viglialoro and Viglialoro ${ }^{20,21}$ for other choices of the source).

Further, let us mention different studies dealing with some specific parabolic-parabolic versions of system (1), defined in a convex smooth and bounded domain $\Omega$ of $\mathbb{R}^{n}, n \geq 1$, endowed with homogeneous Neumann boundary conditions, and under the choice $m=\alpha=1$. Specifically, it has been established that when $\chi(v)=1$ system (1) admits global weak solutions for $k \in \mathbb{R}$ and arbitrarily small values of $\mu>0$ (see Lankeit ${ }^{22}$ ). On the other hand, if $g$ generalises the logistic source (i.e. it verifies $g(0) \geq 0$ and $g(s) \leq k-\mu s^{2}$, for $s \geq 0$ ), $\chi(v)=\chi>0$ and $\mu$ is big enough then the system possesses a unique bounded and global-in-time classical solution. ${ }^{23}$ Additionally, in the case $\chi(v)=\chi>0$, but with source term $g(s) \simeq k-\mu s^{r}$, for $s \geq 0$, and with some $r>1$, global existence of very weak solutions, as well their boundedness properties and long time behaviour are discussed in other works. ${ }^{24-26}$

Finally, in order to better define the purpose of this present investigation, let us frame model (1), in its parabolic-elliptic representation, in the existent literature. For $m=\alpha=1$ and $\chi(v)=\chi>0$, it is proved in Tello and Winkler ${ }^{27}$ that when $\mu>(n-2) \chi / n$ the solutions are globally bounded, whilst for $m \in \mathbb{R}, \alpha=1$ and $\chi(v)=\chi>0$ the same result is achieved in Cao and Zheng ${ }^{28}$ under the assumption $\mu>\left(1-2 /\left(n(2-m)_{+}\right)\right) \chi$. In Zheng, ${ }^{29}$ the author formulates problem (1) 
under the assumptions $m \geq 1, \alpha>0$ and $\chi(v)=\chi>0$, and with a more general expression for the logistic absorption: $k u-\mu u^{r}$, with $r>1$. It is concluded that the coefficient $\mu$ does not take part for the boundedness of the solution when $\alpha<\max \{r, m+2 / n\}-1$, whilst it does for $\alpha+1=r$. Even more, global-in-time existence and uniform-in-time boundedness of classical and weak solutions to (1), with both non-degenerate and degenerate diffusions, have been studied in Wang et al and Xie and Zhaoyin. ${ }^{30,31}$ Nevertheless, as far as we know, and in contrast to models without the logistic source, the question about the existence of a critical exponent, involving the parameters defining the diffusion, the sensitivity and the source capable of warranting, or excluding, the existence of unbounded solutions, is still open.

In accordance with these premises, this contribution aims to present global existence and boundedness of classical solutions to problem (1) when the ranges for the parameters $m$ and $\alpha$ are different from those discussed above. In addition, this report introduces the function $\chi(v)$ in the cross diffusion term, which is not constant but a function from $C^{2}([0, \infty))$ and satisfies for any $v \geq 0$ the growth condition

$$
\chi(v) \leq \frac{\chi_{0}}{(1+a v)^{b}} \quad \text { with some } \quad \chi_{0}>0 \quad \text { and } \quad a \geq 0 \text { and } \quad b>0 .
$$

To be precise, under proper assumptions on the data our mains assertions are summarised in $\S 4$ and claim:

- for $m \in \mathbb{R}, k$ positive and $\alpha<1$, problem (1) with $\chi(v)$ obeying (3) admits a unique globally bounded solution for any $\mu>0$ and any nonnegative and sufficiently regular $u_{0}$ (Theorem 4.3);

- for $m \in \mathbb{R}, k$ positive and $\alpha=1$, problem (1) with $\chi(v)$ obeying (3) admits a unique globally bounded solution for $\mu>0$ sufficiently large and any nonnegative and sufficiently regular $u_{0}$ (Corollary 4.1 ).

The theoretical results presented here are investigated numerically in $\S 5$. Specifically, parameter sweeps of $m, \alpha$, and $n$ are used to detect critical exponents which delineate the regions in which $u$ is, and is not, bounded. Critically, although the inequalities on $\alpha$, herein derived, and from Zheng, ${ }^{29}$ ensure the boundedness of $u$, regardless the size of the dampening term, $\mu$, in the logistic source, these inequalities are not tight. Explicitly, we are able to violate them and still produce bounded simulations. Finally, we consider the influence of $b$ on the solution through simulated parameter sweeps over the interval $b \in[0,2]$.

\section{2 | FIXING SOME PARAMETERS}

In the following Lemma, used throughout the paper to prove the main conclusions, we adjust some parameters, which are necessary in our logical steps.

Lemma 2.1. For any $q_{1}>n+2, q_{2}>(n+2) / 2$ and $m, \alpha \in \mathbb{R}$ with $\alpha \leq 1$ let

$$
\bar{p}:=\max \left\{\begin{array}{c}
\frac{n}{2}(1-m) \\
1-\alpha \\
q_{1} \\
1-m \frac{(n+1) q_{1}-(n+2)}{q_{1}-(n+2)} \\
1-\frac{m}{1-\frac{n}{n+2} \frac{q_{2}}{q_{2}-1}}
\end{array}\right\} .
$$

Then these relations hold:

$$
\begin{gathered}
p>\frac{n}{2}(1-m) \text { forall } p>\bar{p} \text { and } n \in \mathbb{N}, \\
0<\frac{\frac{n}{2}(m+p-1)\left(1-\frac{1}{p}\right)}{1-\frac{n}{2}+\frac{n}{2}(m+p-1)}<1 \text { for all } p>\bar{p} \text { and } n \in \mathbb{N}, \\
0<\frac{p+\alpha}{p+1}<1 \text { forall } p>\bar{p}, \quad \alpha \neq 1 \text { and } n \in \mathbb{N},
\end{gathered}
$$




$$
\begin{gathered}
p+\alpha-1>0 \text { for all } p>\bar{p} \text { and } n \in \mathbb{N}, \\
p>q_{1} \text { for all } p>\bar{p} \text { and } n \in \mathbb{N}, \\
p>1-m \frac{(n+1) q_{1}-(n+2)}{q_{1}-(n+2)} \text { for all } p>\bar{p} \text { and } n \in \mathbb{N}, \\
p>1-\frac{m}{1-\frac{n}{n+2} \frac{q_{2}}{q_{2}-1}} \text { for all } p>\bar{p} \text { and } n \in \mathbb{N} .
\end{gathered}
$$

Proof. From the expression of $\bar{p}$, we have that

$$
\frac{m+\bar{p}-1}{2} \geq \frac{m+\bar{p}-1}{2 \bar{p}}, \quad \frac{m+\bar{p}-1}{2 \bar{p}}>\frac{n-2}{2 n} \text { and } \bar{p}>\frac{n}{2}(1-m)
$$

therefore,

$$
1-\frac{n}{2}+\frac{n}{2}(m+p-1)>0
$$

and, thus, (6) is attained. In addition, the remaining inequalities are clearly verified for any $p>\bar{p}$ once $\bar{p}$ is defined as in (4).

\section{3 | EXISTENCE OF LOCAL-IN-TIME SOLUTIONS AND THEIR PROPERTIES}

Our first result concerns local-in-time existence of classical solutions to system (1). The proof of the result is obtained by well-established methods involving standard parabolic-elliptic regularity theory and an appropriate fixed point framework. ${ }^{13,16,32}$

Lemma 3.1. Let $\Omega$ be a smooth and bounded domain of $\mathbb{R}^{n}$, with $n \geq 1$. For given $m, \alpha \in \mathbb{R}$, and $k$, $\mu$ positive, let us assume that $\chi \in C^{2}\left([0, \infty)\right.$ ) satisfies relation (3). Then for any nonnegative function $u_{0} \in W^{1, \infty}(\Omega)$, problem (1) admits a unique local-in-time classical solution

$$
(u, v) \in\left(C\left(\left[0, T_{\max }\right) ; W^{1, \infty}(\Omega)\right) \cap C^{2,1}\left(\bar{\Omega} \times\left(0, T_{\max }\right)\right)\right)^{2},
$$

where $T_{\max }$ denotes the maximal existence time. Moreover, we have

$$
u \geq 0 \text { and } v \geq 0 \text { in } \Omega \times\left(0, T_{\max }\right) \text {, }
$$

and if $T_{\max }<\infty$ then

$$
\limsup _{t>T_{\max }}\|u(\cdot, t)\|_{L^{\infty}(\Omega)}=\infty .
$$

Proof. Uniqueness. By absurdity let $\left(u_{1}, v_{1}\right)$ and $\left(u_{2}, v_{2}\right)$ be 2 nonnegative different solutions of $(1)$ in $\Omega \times\left(0, T_{\max }\right)$ with the same initial data, that is $u_{1}(\cdot, 0)=u_{2}(\cdot, 0)$ and $v_{1}(\cdot, 0)=v_{2}(\cdot, 0)$. In such circumstances $v_{1}-v_{2}$ solves

$$
-\Delta\left(v_{1}-v_{2}\right)+\left(v_{1}-v_{2}\right)=u_{1}-u_{2} .
$$

Differentiating Equation 13 with respect to $t$, multiplying by a factor $v_{1}-v_{2}$ and integrating over space, yield, for all $t \in\left(0, T_{0}\right)$ with $T_{0}<T_{\max }$, 


$$
\begin{aligned}
& \frac{1}{2} \frac{d}{d t} \int_{\Omega}\left|\nabla\left(v_{1}-v_{2}\right)\right|^{2}+\frac{1}{2} \frac{d}{d t} \int_{\Omega}\left(v_{1}-v_{2}\right)^{2} \\
= & \int_{\Omega}\left(u_{1}-u_{2}\right)_{t}\left(v_{1}-v_{2}\right)-\int_{\Omega} \Delta\left(v_{1}-v_{2}\right)_{t}\left(v_{1}-v_{2}\right)+\left(v_{1}-v_{2}\right)\left(v_{1}-v_{2}\right)_{t}, \\
= & \int_{\Omega}\left(u_{1}-u_{2}\right)_{t}\left(v_{1}-v_{2}\right), \\
= & -\int_{\Omega} \nabla\left(\frac{\left(u_{1}+1\right)^{m}}{m}-\frac{\left(u_{2}+1\right)^{m}}{m}\right) \cdot \nabla\left(v_{1}-v_{2}\right) \\
& +\int_{\Omega}\left(\chi\left(v_{1}\right) u_{1}\left(u_{1}+1\right)^{\alpha-1} \nabla v_{1}-\chi\left(v_{2}\right) u_{2}\left(u_{2}+1\right)^{\alpha-1} \nabla v_{2}\right) \cdot \nabla\left(v_{1}-v_{2}\right) \\
& +\int_{\Omega}\left(k u_{1}-\mu u_{1}^{2}-k u_{2}+\mu u_{2}^{2}\right)\left(v_{1}-v_{2}\right) .
\end{aligned}
$$

Let us set

$$
\begin{aligned}
& s_{1}:=\min \left\{\left\|u_{1}\right\|_{L^{\infty}\left(\Omega \times\left(0, T_{0}\right)\right)},\left\|u_{2}\right\|_{L^{\infty}\left(\Omega \times\left(0, T_{0}\right)\right)}\right\}, \\
& s_{2}:=\max \left\{\left\|u_{1}\right\|_{L^{\infty}\left(\Omega \times\left(0, T_{0}\right)\right)},\left\|u_{2}\right\|_{L^{\infty}\left(\Omega \times\left(0, T_{0}\right)\right)}\right\} .
\end{aligned}
$$

The Mean Value Theorem applied to the function $s \mapsto(s+1)^{m} / m$ in the interval $\left[s_{1}, s_{2}\right]$ infers for some $\bar{s} \in\left(s_{1}, s_{2}\right)$

$$
\frac{\left(s_{1}+1\right)^{m}}{m}-\frac{\left(s_{2}+1\right)^{m}}{m}=(\bar{s}+1)^{m-1}\left(s_{1}-s_{2}\right),
$$

so that $\left(s_{1}+1\right)^{m-1} \leq(\bar{s}+1)^{m-1} \leq\left(s_{2}+1\right)^{m-1}$. In light of this, by virtue of the Young inequality, an integration by parts and relation (13), there exists some positive $C_{1}$ depending on $T_{0}$ and belonging to the interval $\left[\left(s_{1}+1\right)^{m-1},\left(s_{2}+1\right)^{m-1}\right]$ such that

$$
\begin{aligned}
I_{1} & :=-\int_{\Omega} \nabla\left(\frac{\left(u_{1}+1\right)^{m}}{m}-\frac{\left(u_{2}+1\right)^{m}}{m}\right) \cdot \nabla\left(v_{1}-v_{2}\right) \\
& =\int_{\Omega}\left(\frac{\left(u_{1}+1\right)^{m}}{m}-\frac{\left(u_{2}+1\right)^{m}}{m}\right) \Delta\left(v_{1}-v_{2}\right) \\
& =\int_{\Omega}\left(\frac{\left(u_{1}+1\right)^{m}}{m}-\frac{\left(u_{2}+1\right)^{m}}{m}\right)\left(v_{1}-v_{2}\right) \\
& -\int_{\Omega}\left(\frac{\left(u_{1}+1\right)^{m}}{m}-\frac{\left(u_{2}+1\right)^{m}}{m}\right)\left(u_{1}-u_{2}\right) \\
& =C_{1} \int_{\Omega}\left(u_{1}-u_{2}\right)\left(v_{1}-v_{2}\right)-C_{1} \int_{\Omega}\left(u_{1}-u_{2}\right)^{2} \\
& \leq \frac{C_{1}}{2} \int_{\Omega}\left(v_{1}-v_{2}\right)^{2}-\frac{C_{1}}{2} \int_{\Omega}\left(u_{1}-u_{2}\right)^{2} .
\end{aligned}
$$

Next, we define

$$
I_{2}:=\int_{\Omega}\left(\chi\left(v_{1}\right) u_{1}\left(u_{1}+1\right)^{\alpha-1} \nabla v_{1}-\chi\left(v_{2}\right) u_{2}\left(u_{2}+1\right)^{\alpha-1} \nabla v_{2}\right) \cdot \nabla\left(v_{1}-v_{2}\right)
$$

In view of the regularity of the function $\xi \mapsto \xi(\xi+1)^{\alpha-1}$, with $\xi \geq 0$, and also well-known elliptic theory results, we have that for some $C_{2}=C_{2}\left(T_{0}\right)$

$$
\left\{\begin{array}{l}
\left|u_{1}\left(u_{1}+1\right)^{\alpha-1}-u_{2}\left(u_{2}+1\right)^{\alpha-1}\right| \leq C_{2}\left|u_{1}-u_{2}\right| \text { in } \Omega \times\left(0, T_{0}\right), \\
u_{2}\left(u_{2}+1\right)^{\alpha-1} \leq C_{2} \quad \text { in } \Omega \times\left(0, T_{0}\right) \\
\left|\nabla v_{1}\right| \leq C_{2} \text { in } \Omega \times\left(0, T_{0}\right)
\end{array}\right.
$$

Subsequently, these relations, in conjunction with the Hölder inequality and the bound for $\chi(v)$, infer 


$$
\begin{aligned}
I_{2}^{2} \leq & \chi_{0}^{2} \int_{\Omega}\left(\left|u_{1}\left(u_{1}+1\right)^{\alpha-1} \nabla v_{1}-u_{2}\left(u_{2}+1\right)^{\alpha-1} \nabla v_{2}\right|^{2}\right) \int_{\Omega}\left|\nabla\left(v_{1}-v_{2}\right)\right|^{2}, \\
\leq & \chi_{0}^{2} \int_{\Omega}\left|u_{1}\left(u_{1}+1\right)^{\alpha-1}-u_{2}\left(u_{2}+1\right)^{\alpha-1}\right|^{2}\left|\nabla v_{1}\right|^{2} \int_{\Omega}\left|\nabla\left(v_{1}-v_{2}\right)\right|^{2} \\
& +\chi_{0}^{2} \int_{\Omega}\left|u_{2}\left(u_{2}+1\right)^{\alpha-1} \nabla\left(v_{1}-v_{2}\right)\right|^{2} \int_{\Omega}\left|\nabla\left(v_{1}-v_{2}\right)\right|^{2}, \\
& \leq \chi_{0}^{2} C_{2}^{4} \int_{\Omega}\left(u_{1}-u_{2}\right)^{2} \int_{\Omega}\left|\nabla\left(v_{1}-v_{2}\right)\right|^{2}+\chi_{0}^{2} C_{2}^{2}\left(\int_{\Omega}\left|\nabla\left(v_{1}-v_{2}\right)\right|^{2}\right)^{2} .
\end{aligned}
$$

Hence, using the inequality $(A+B)^{\frac{1}{2}} \leq A^{\frac{1}{2}}+B^{\frac{1}{2}}$, which is valid for any $A, B \geq 0$, and then the Young inequality, we have for some $\rho_{1}>0$

$$
I_{2} \leq \frac{\chi_{0} C_{2}^{2} \rho_{1}}{2} \int_{\Omega}\left(u_{1}-u_{2}\right)^{2}+\left(\frac{\chi_{0} C_{2}^{2}}{2 \rho_{1}}+C_{2} \chi_{0}\right) \int_{\Omega}\left|\nabla\left(v_{1}-v_{2}\right)\right|^{2}
$$

Additionally, application of the Mean Value Theorem, the Young inequality and the boundedness of $u_{1}$ and $u_{2}$ in $\Omega \times\left(0, T_{0}\right)$ provide some $C_{3}=C_{3}\left(T_{0}\right)>0$

$$
\int_{\Omega}\left(k u_{1}-\mu u_{1}^{2}-k u_{2}+\mu u_{2}^{2}\right)\left(v_{1}-v_{2}\right) \leq \frac{C_{3} \rho_{2}}{2} \int_{\Omega}\left(u_{1}-u_{2}\right)^{2}+\frac{C_{3}}{2 \rho_{2}} \int_{\Omega}\left(v_{1}-v_{2}\right)^{2},
$$

with some $\rho_{2}>0$.

Choosing $\rho_{1}=C_{1} /\left(2 \chi_{0} C_{2}^{2}\right)$ and $\rho_{2}=C_{1} /\left(2 C_{3}\right)$, and successively inserting into Equation 14 relations (15), (16), and (17), we arrive for some computable constant $C_{4}\left(T_{0}\right)>0$ at the initial problem

$$
\frac{d}{d t} \mathcal{F} \leq C_{4} \mathcal{F} \quad t \in\left(0, T_{0}\right), \quad \mathcal{F}(0)=0
$$

where $\mathcal{F}(t):=\int_{\Omega}\left|\nabla\left(v_{1}-v_{2}\right)\right|^{2}+\int_{\Omega}\left(v_{1}-v_{2}\right)^{2}$. Since Equation 18 admits the unique solution $\mathcal{F} \equiv 0$ on $\left(0, T_{0}\right)$, due to the arbitrary of $T_{0}$, we attain $v_{1}=v_{2}$ on $\left(0, T_{\max }\right)$ and hence $u_{1}=u_{2}$.

Existence.

For any $T \in(0,1)$ and $R:=\left\|u_{0}\right\|_{L^{\infty}(\Omega)}+1$, let us consider the Banach space $X:=C^{0}(\bar{\Omega} \times[0, T])$ and its closed subset

$$
S:=\left\{u \in X \mid u \geq 0 \text { in } \bar{\Omega} \times[0, T] \text { and }\|u(\cdot, t)\|_{L^{\infty}(\Omega)} \leq R \text { for all } t \in(0, T)\right\}
$$

For $\bar{u} \in S$, let $v$ be the solution of

$$
\begin{cases}0=\Delta v-v+\bar{u} & \Omega \times(0, T) \\ \frac{\partial v}{\partial v}=0 & \partial \Omega \times(0, T),\end{cases}
$$

and, in turn, let $u$ be the solution of

$$
\begin{cases}u_{t}-\nabla \cdot(\bar{u}+1)^{m-1} \nabla u=\nabla \cdot u(\bar{u}+1)^{\alpha-1} \chi(v) \nabla v+k u-\mu u^{2} & \Omega \times(0, T), \\ \frac{\partial u}{\partial v}=0 & \partial \Omega \times(0, T), \\ u(x, 0)=u_{0}(x) \geq 0 & \Omega .\end{cases}
$$

In agreement with these statements, we shall show that for appropriate small $T, \Phi: S \rightarrow S$ defined by $\Phi(\bar{u})=u$ is a compact map such that $\Phi(S) \subset S$. Subsequently, due to the topological properties of $S$, the Schauder fixed point theorem shows that there exists $u \in S$ such that $\Phi(u)=u$.

First, we observe that for some $\delta \in(0,1)$ the elliptic regularity theory (theorem 8.34 of Gilbarg and Trudinger ${ }^{33}$ ) ensures the existence of a unique solution $v(\cdot, t)$ of problem $(19)$ which belongs to $C^{1+\delta}(\Omega)$. Moreover, for some positive constant $c$ (which until the end of this proof might change line by line), we have from elliptic regularity results that for all $t \in(0, T)$ 


$$
\|v(\cdot, t)\|_{W^{2, p}(\Omega)} \leq c\|\bar{u}(\cdot, t)\|_{L^{p}(\Omega)} ;
$$

in particular, $\nabla v \in W^{1, p}(\Omega)$ for all $t \in(0, T)$ and subsequently the Sobolev embedding theorem with $p>n$ ensures that $\nabla v \in L^{\infty}(\Omega)$ for all $t \in(0, T)$. Additionally, since $\bar{u} \in S$, for any $m \in \mathbb{R}$ and $t \in(0, T)$, we have that $\left\|(\bar{u}+1)^{m-1}\right\|_{L^{\infty}(\Omega)} \leq$ $c$, so that the classical parabolic regularity (theorem V.1.1. of Ladyženskaja et al ${ }^{34}$ ) applied to problem (20) implies that $u \in C^{\delta_{1}, \frac{\delta_{1}}{2}}(\Omega \times(0, T))$, for some $\delta_{1} \in(0,1)$. Hence,

$$
u(\cdot, t) \leq u_{0}+c t^{\frac{\delta_{1}}{2}} \quad \text { for all } t \in(0, T)
$$

and thereafter

$$
\max _{t \in[0, T]}\|u(\cdot, t)\|_{L^{\infty}(\Omega)} \leq\left\|u_{0}\right\|_{L^{\infty}(\Omega)}+c T^{\frac{\delta_{1}}{2}} .
$$

Subsequently, for $T<c^{\frac{-\delta_{1}}{2}}$, we also deduce that

$$
\max _{t \in[0, T]}\|u(\cdot, t)\|_{L^{\infty}(\Omega)} \leq\left\|u_{0}\right\|_{L^{\infty}(\Omega)}+1=R .
$$

On the other hand, $k u-\mu u^{2}$ is 0 for $u=0$, so that the parabolic comparison principle ensures that $u$ is nonnegative; hence, $\Phi$ maps $S$ into itself, compactly since $C^{\delta_{1}}, \frac{\delta_{1}}{2}(\Omega \times(0, T)) \hookrightarrow X$. Let $u$ be the fix point of $\Phi$; by using the elliptic regularity theory to problem (19) and the parabolic one to problem (20) (explicitly Theorem V.6.1. of Ladyženskaja et $\left.\mathrm{al}^{34}\right)$, we have that $u, v \in C^{2+\delta_{1}, 1+\frac{\delta_{1}}{2}}(\Omega \times[\tau, T])$, for any $\tau \in(0, T)$. Further, with $u$ being nonnegative the elliptic comparison principle applied to (19) implies that $v$ is nonnegative as well. Moreover, by standard arguments the solution may be prolonged in the interval $\left[0, T_{\max }\right.$ ), with $T_{\max } \leq \infty, T_{\max }$ being finite if and only if (12) holds.

Lemma 3.2. Let $\Omega$ be a smooth and bounded domain of $\mathbb{R}^{n}$, with $n \geq 1$. For any nonnegative function $u_{0} \in W^{1, \infty}(\Omega)$, let $(u, v)$ be the local-in-time classical solution of problem (1) provided by Lemma 3.1. Then we have

$$
\int_{\Omega} u(\cdot, t) \leq M \text { for all } t \in\left(0, T_{\max }\right)
$$

where $M=\max \left\{k|\Omega| / \mu, \int_{\Omega} u_{0}\right\}$

Proof. Taking into consideration the no-flux boundary conditions for problem (1), an integration of its first equation over $\Omega$ and an application of the Hölder inequality provide

$$
\frac{d}{d t} \int_{\Omega} u=k \int_{\Omega} u-\mu \int_{\Omega} u^{2} \leq k \int_{\Omega} u-\frac{\mu}{|\Omega|}\left(\int_{\Omega} u\right)^{2} \text { for all } t \in\left(0, T_{\max }\right),
$$

so that (21) is a consequence of a comparison argument.

\section{4 | A PRIORI ESTIMATES AND PROOF OF THE MAIN RESULTS}

In this section, we shall gain some uniform bound for $u$, by bounding $\|u\|_{L^{p}(\Omega)}$, for $p$ sufficiently large and on the whole interval $\left(0, T_{\max }\right)$ with a suitable positive and time independent constant. This constant is attained by establishing an absorptive differential inequality for $\Phi(t)=\int_{\Omega}(u+1)^{p}$ and using comparison principles.

Lemma 4.1. Let $\Omega$ be a smooth and bounded domain of $\mathbb{R}^{n}$, with $n \geq 1$. For $\alpha<1$ and any nonnegative function $u_{0} \in W^{1, \infty}(\Omega)$, let $(u, v)$ be the local-in-time classical solution of problem (1) provided by Lemma 3.1. Then, for any $p>\bar{p}$, $\bar{p}$ being the constant given in (4), and $\varepsilon$ positive real number, we have

$$
\begin{aligned}
& \frac{1}{p} \frac{d}{d t} \int_{\Omega}(u+1)^{p}+\frac{4(p-1)}{(m+p-1)^{2}} \int_{\Omega}\left|\nabla(u+1)^{\frac{m+p-1}{2}}\right|^{2} \leq \\
& +\left(\frac{\varepsilon}{p} \chi_{0}-\mu\right) \int_{\Omega}(u+1)^{p+1}+c_{0},
\end{aligned}
$$


where

$$
\left\{\begin{array}{l}
C_{1}(\varepsilon)=\frac{1-\alpha}{p+1}\left(\frac{\varepsilon(p+1)}{2 p(p+\alpha)} \frac{p+\alpha-1}{p-1}\right)^{\frac{p+\alpha}{\alpha-1}}, \\
C_{2}(\varepsilon)=\frac{1}{p+1}\left(\frac{\varepsilon(p+1) \chi_{0}}{2(2 \mu+k) p^{2}}\right)^{-p}, \\
c_{0}=\frac{p-1}{p+\alpha-1} \chi_{0} C_{1}(\varepsilon)|\Omega|+(2 \mu+k) C_{2}(\varepsilon)|\Omega| .
\end{array}\right.
$$

Proof. For $\bar{p}$ as in (4), let $p>\bar{p}$; testing the first equation of problem (1) by $p(u+1)^{p-1}$, using its boundary conditions and relation (3) provide

$$
\begin{aligned}
\frac{1}{p} \frac{d}{d t} \int_{\Omega}(u+1)^{p}= & \int_{\Omega}(u+1)^{p-1} u_{t} \leq-(p-1) \int_{\Omega}(u+1)^{p+m-3}|\nabla u|^{2} \\
& +(p-1) \chi_{0} \int_{\Omega}(u+1)^{p+\alpha-2} \nabla u \cdot \nabla v \\
& +k \int_{\Omega} u(u+1)^{p-1}-\mu \int_{\Omega} u^{2}(u+1)^{p-1}
\end{aligned}
$$

on $\left(0, T_{\max }\right)$. Now, taking into consideration that from the second equation of $(1)$, we have that

$$
-(u+1)^{p+\alpha-1} \Delta v=-(u+1)^{p+\alpha-1}(v-u) \leq(u+1)^{p+\alpha} \quad t \in\left(0, T_{\max }\right),
$$

through an integration by parts we infer that, in view of the fact that $p+\alpha-1>0$ for all $p>\bar{p}$ (see relation (8)

$$
\begin{aligned}
(p-1) \chi_{0} \int_{\Omega}(u+1)^{p+\alpha-2} \nabla u \cdot \nabla v & =\frac{(p-1) \chi_{0}}{p+\alpha-1} \int_{\Omega} \nabla(u+1)^{p+\alpha-1} \cdot \nabla v, \\
& =-\frac{(p-1) \chi_{0}}{p+\alpha-1} \int_{\Omega}(u+1)^{p+\alpha-1} \Delta v \\
& \leq \frac{(p-1) \chi_{0}}{p+\alpha-1} \int_{\Omega}(u+1)^{p+\alpha}
\end{aligned}
$$

for all $t \in\left(0, T_{\max }\right)$. Since from (7), we have that $0<(p+\alpha) /(p+1)<1$, an application of the Young inequality gives for any $\varepsilon$ on $\left(0, T_{\max }\right)$

$$
\int_{\Omega}(u+1)^{p+\alpha} \leq \frac{p+\alpha-1}{p-1} \frac{\varepsilon}{2 p} \int_{\Omega}(u+1)^{p+1}+C_{1}(\varepsilon)|\Omega| .
$$

As to the contribution from the logistic source, for all $t \in\left(0, T_{\max }\right)$ we can write

$$
k \int_{\Omega} u(u+1)^{p-1}-\mu \int_{\Omega} u^{2}(u+1)^{p-1} \leq k \int_{\Omega}(u+1)^{p}-\mu \int_{\Omega}(u+1)^{p+1}+2 \mu \int_{\Omega}(u+1)^{p},
$$

where we have used the inequality $-u^{2} \leq-(u+1)^{2}+2(u+1)$. Successively, the Young inequality enables us to deduce that on $\left(0, T_{\max }\right)$

$$
(2 \mu+k) \int_{\Omega}(u+1)^{p} \leq \frac{\varepsilon \chi_{0}}{2 p} \int_{\Omega}(u+1)^{p+1}+(2 \mu+k) C_{2}(\varepsilon)|\Omega|
$$

Taking into account that

$$
(p-1) \int_{\Omega}(u+1)^{p+m-3}|\nabla u|^{2}=\frac{4(p-1)}{(m+p-1)^{2}} \int_{\Omega}\left|\nabla(u+1)^{\frac{m+p-1}{2}}\right|^{2},
$$

our thesis is justified once Equations 23 to 28 are collected together. 
Lemma 4.2. Let $\Omega$ be a smooth and bounded domain of $\mathbb{R}^{n}$, with $n \geq 1$. For $\alpha<1$ and any nonnegative function $u_{0} \in W^{1, \infty}(\Omega)$, let $(u, v)$ be the local-in-time classical solution of problem (1) provided by Lemma 3.1. Then there exists a positive constant $L_{1}$ such that for any $p>\bar{p}$

$$
\int_{\Omega}(u+1)^{p} \leq L_{1} \quad \text { for all } t \in\left(0, T_{\max }\right) .
$$

Proof. For any $p>\bar{p}$, let us set $\Phi(t)=\frac{1}{p} \int_{\Omega}(u+1)^{p}$. By choosing the constant $\varepsilon$ introduced above in the interval $\left(0, \mu p / \chi_{0}\right]$, relation $(22)$ is reduced to

$$
\Phi^{\prime}(t)+\frac{4(p-1)}{(m+p-1)^{2}} \int_{\Omega}\left|\nabla(u+1)^{\frac{m+p-1}{2}}\right|^{2} \leq c_{0} .
$$

Now, the Gagliardo-Nirenberg inequality, in conjunction with

$$
(A+B)^{k} \leq 2^{k}\left(A^{k}+B^{k}\right),
$$

valid for any $A, B \geq 0$ and $k>0$, infer that for

$$
0<\theta_{1}=\frac{n \frac{m+p-1}{2}\left(1-\frac{1}{p}\right)}{1-\frac{n}{2}+n \frac{m+p-1}{2}}<1 \quad \text { (recall (6) of Lemma 2.1), }
$$

the relation

$$
\begin{aligned}
\int_{\Omega}(u+1)^{p} & =\left\|(u+1)^{\frac{m+p-1}{2}}\right\|_{L^{\frac{2 p}{m+p-1}}(\Omega)}^{\frac{2 p}{m+1}} \\
& \leq c_{1}\left\|\nabla(u+1)^{\frac{m+p-1}{2}}\right\|_{L^{2}(\Omega)}^{\frac{2 p}{m+p-1} \theta_{1}}\left\|(u+1)^{\frac{m+p-1}{2}}\right\|_{L^{\frac{2}{m+p-1}}(\Omega)}^{\left(1-\theta_{1}\right) \frac{2 p}{m+p-1}} \\
& +c_{1}\left\|(u+1)^{\frac{m+p-1}{2}}\right\|_{\substack{\frac{2 p}{m+p-1} \\
L^{\frac{2}{m+p-1}}(\Omega)}}^{\frac{2}{m-1}}
\end{aligned}
$$

is verified with $c_{1}=\left(2 C_{G N}\right)^{\frac{2 p}{m+p-1}}$. Considering the bound from inequality (21) and introducing $c_{2}=$ $c_{1} \max \left\{(M+|\Omega|)^{\left(1-\theta_{1}\right) p},(M+|\Omega|)^{p}\right\}$, we observe that inequality (32) can be written as

$$
\int_{\Omega}(u+1)^{p} \leq c_{2}\left(\int_{\Omega}\left|\nabla(u+1)^{\frac{m+p-1}{2}}\right|^{2}\right)^{\frac{p \theta_{1}}{m+p-1}}+c_{2} \quad t \in\left(0, T_{\max }\right) .
$$

As a consequence of all of the above, by making first use of inequality (31) in (33) and then inserting the result into (30), we obtain that $\Phi$ verifies this initial problem

$$
\left\{\begin{array}{l}
\Phi^{\prime}(t) \leq c_{3}-c_{4} \Phi^{\frac{m+p-1}{p \theta_{1}}}(t) \quad t \in\left(0, T_{\max }\right) \\
\Phi(0)=\int_{\Omega}\left(u_{0}+1\right)^{p}
\end{array}\right.
$$

with

$$
c_{3}=c_{0}+\frac{4(p-1)}{(m+p-1)^{2}} \quad \text { and } \quad c_{4}=\frac{4(p-1)}{(m+p-1)^{2}}\left(\frac{2 c_{2}}{p}\right)^{-\frac{m+p-1}{p \theta_{1}}} .
$$

Consequently, an application of a comparison principle implies that

$$
\Phi(t) \leq \max \left\{\Phi(0),\left(\frac{c_{3}}{c_{4}}\right)^{\frac{p \theta_{1}}{m+p-1}}\right\}:=L_{1} \text { for all } t \in\left(0, T_{\max }\right),
$$

concluding the proof.

After these preparations, we can prove the first of our two results. 
Theorem 4.3. Let $\Omega$ be a smooth and bounded domain of $\mathbb{R}^{n}$, with $n \geq 1$. For given $m \in \mathbb{R}, k$, $\mu$ positive and $\alpha<1$, let us assume that $\chi \in C^{2}\left([0, \infty)\right.$ ) satisfies relation (3). Then for any nonnegative function $u_{0} \in W^{1, \infty}(\Omega)$, problem (1) admits a unique global classical solution $(u, v)$. Moreover, both $u$ and $v$ are bounded in $\Omega \times(0, \infty)$.

Proof. Let $(u, v)$ be the local-in-time classical solution of system (1) emanating from any nonnegative function $u_{0} \in$ $W^{1, \infty}(\Omega)$ and provided by Lemma 3.1. Hereafter, coherent with the nomenclature used by Tao and Winkler, $u$ also classically solves the problem (A.1) of appendix A of Tao and Winkler ${ }^{35}$ in $\Omega \times\left(0, T_{\max }\right)$ with

$$
D(x, t, u)=(u+1)^{m-1}, \quad f(x, t)=-u(u+1)^{\alpha-1} \chi(v) \nabla v, \quad g(x, t)=\frac{k^{2}}{4 \mu} .
$$

For $\bar{p}$ defined in (4) of Lemma 2, Lemma 4.2 directly applies to warrant that for any $p>\bar{p}$ holds that

$$
u \in L^{\infty}\left(\left(0, T_{\max }\right) ; L^{p}(\Omega)\right) .
$$

In particular, we deduce that (A.2) to (A.5), the second inclusion of (A.6) for any choice of $q_{2}$ and (A.7) with $p_{0}=p$, are verified on $\left(0, T_{\max }\right)$. From relation (34), elliptic regularity results applied to the equation $-\Delta v+v=u$ imply $v \in L^{\infty}\left(\left(0, T_{\max }\right) ; W^{2, p}(\Omega)\right)$ and, hence, $\nabla v \in L^{\infty}\left(\left(0, T_{\max }\right) ; W^{1, p}(\Omega)\right)$. In particular, due to the Sobolev embeddings, we infer $\nabla v \in L^{\infty}\left(\left(0, T_{\max }\right) ; L^{\infty}(\Omega)\right)$, since from the definition of $\bar{p}$, we also have $p>n$ (recall (9). Consequently, as to the first condition of (A.6), bounds (3) and (29), and the fact that $\alpha q_{1}<q_{1}<p$, show, through the Hölder inequality with exponents $\alpha q_{1} / p$ and $1-\alpha q_{1} / p$ that this bound holds on $\left(0, T_{\max }\right)$ :

$$
\begin{aligned}
\int_{\Omega}|f|^{q_{1}} & \leq \int_{\Omega}(u+1)^{\alpha q_{1}}|\chi(v)|^{q_{1}}|\nabla v|^{q_{1}} \\
& \leq \chi_{0}^{q_{1}}\|\nabla v(\cdot, t)\|_{L^{\infty}(\Omega)}^{q_{1}}|\Omega|^{\frac{p-\alpha q_{1}}{p}}\left(\int_{\Omega}(u+1)^{p}\right)^{\frac{\alpha q_{1}}{p}} .
\end{aligned}
$$

Hence, we also attain that for any $n \geq 1$

$$
f \in L^{\infty}\left(\left(0, T_{\max }\right) ; L^{q_{1}}(\Omega)\right), \text { with } q_{1}>n+2 .
$$

Moreover, by virtue of expressions (10), (11), and (5), relations (A.8), (A.9) for $q_{2}>(n+2) / 2$, and (A.10) of Lemma A.1. of Tao and Winkler ${ }^{35}$ are also valid, so we get that for some $L_{2}>0$

$$
\|u(\cdot, t)\|_{L^{\infty}(\Omega)} \leq L_{2} \quad \text { for all } t \in\left(0, T_{\max }\right) .
$$

In turn, the extensibility criterion (12) of Lemma 3.1 shows that $T_{\max }=\infty$. Finally, the independence of the obtained estimate with respect to $t \in\left(0, T_{\max }\right)=(0, \infty)$, justified by the main uniform-in-time bound (29), establishes that $u$ is bounded in $(0, \infty)$ and through the second equation of $(1)$ also the uniform bound of $v$ is achieved.

By retracing the proof of Lemma 4.1, we observe that the condition $\alpha<1$ is exactly required in relation (25) to make the Young inequality applicable. For $\alpha=1$, the Young inequality is senseless and the machinery used in the proof above is no longer valid. Nevertheless, obstacles can be circumvented. To be more precise, as a by product of the previous reasoning and through rearranging and manipulating some statements, we can also prove

Corollary 4.1. Let $\Omega$ be a smooth and bounded domain of $\mathbb{R}^{n}$, with $n \geq 1$. For given $m \in \mathbb{R}, k$, $\mu$ positive and $\alpha=1$, let us assume that $\chi \in C^{2}\left([0, \infty)\right.$ ) satisfies relation (3). Then for any nonnegative function $u_{0} \in W^{1, \infty}(\Omega)$, it is possible to find a positive constant $K=K(n, m)$ such that if

$$
\mu>K(n, m) \chi_{0},
$$

problem (1) admits a unique global classical solution $(u, v)$. Moreover, both $u$ and $v$ are bounded in $\Omega \times(0, \infty)$.

Proof. Let $(u, v)$ be the local-in-time classical solution of problem (1) emanating from any nonnegative function $u_{0} \in$ $W^{1, \infty}(\Omega)$; estimate (24) becomes 


$$
\begin{aligned}
(p-1) \chi_{0} \int_{\Omega}(u+1)^{p+\alpha-2} \nabla u \cdot \nabla v & =\frac{(p-1) \chi_{0}}{p} \int_{\Omega} \nabla(u+1)^{p} \cdot \nabla v, \\
& =-\frac{(p-1) \chi_{0}}{p} \int_{\Omega}(u+1)^{p} \Delta v, \\
& \leq \frac{(p-1) \chi_{0}}{p} \int_{\Omega}(u+1)^{p+1} .
\end{aligned}
$$

In view of this, for all $p>\bar{p}, \bar{p}$ given by (4), inequality (22) now reads

$$
\begin{aligned}
\frac{1}{p} \frac{d}{d t} \int_{\Omega}(u+1)^{p} & +\frac{4(p-1)}{(m+p-1)^{2}} \int_{\Omega}\left|\nabla(u+1)^{\frac{m+p-1}{2}}\right|^{2} \leq \\
& +\left(\frac{\tilde{\varepsilon} \chi_{0}}{2 p}+\frac{p-1}{p} \chi_{0}-\mu\right) \int_{\Omega}(u+1)^{p+1}+\tilde{c}_{0},
\end{aligned}
$$

where $\tilde{c}_{0}=(2 \mu+k) C_{2}(\tilde{\varepsilon})$, with $C_{2}=C_{2}(\cdot)$ defined in Lemma 4.1 and $\tilde{\varepsilon}>0$. Now, for any $p>\bar{p}$, if $\mu$ satisfies the following relation

$$
\mu>k(p) \chi_{0}
$$

where

$$
k(p)=1-\frac{1}{p},
$$

we can choose $\tilde{\varepsilon}>0$ such that $0<\tilde{\varepsilon}<2 p\left(\mu-k(p) \chi_{0}\right)$ so that

$$
\frac{\tilde{\varepsilon} \chi_{0}}{2 p}+\frac{p-1}{p} \chi_{0}-\mu<0
$$

and inequality (36) is equivalent to

$$
\Phi^{\prime}(t)+\frac{4(p-1)}{(m+p-1)^{2}} \int_{\Omega}\left|\nabla(u+1)^{\frac{m+p-1}{2}}\right|^{2} \leq \tilde{c}_{0} .
$$

The same remaining steps of Lemma 4.1 show that there exists a positive constant $L_{1}$ such that for any $p>\bar{p}$

$$
\int_{\Omega}(u+1)^{p} \leq L_{1} \quad \text { for all } t \in\left(0, T_{\max }\right) .
$$

Now, let us set $K(n, m)=k(\bar{p})$, where $k(p)$ has been introduced in (38). Since relation (35) is satisfied, we have, by continuity reasons, that there exists $p>\bar{p}$ such that $\mu>k(p) \chi_{0}$. Subsequently, assumption (37) holds so that bound (29) implies that

$$
u \in L^{\infty}\left(\left(0, T_{\max }\right) ; L^{p}(\Omega)\right),
$$

and we conclude as in the proof of Theorem 4.3.

Remark 1. By taking $m=1$ in (1) and $b=0$ in (3), for the limit case $\alpha=1$ it is seen by its expression in Lemma 2 that $\bar{p}=q_{1}$. In this sense, since assumption (38) has to hold for all $p>\bar{p}>n+2$, the optimal condition for the boundedness established in (37) corresponds to the infimum of $k(p)$ and it is hence rewritten as

$$
\mu>\frac{n+1}{n+2} \chi_{0} .
$$

We observe that even though the criterion herein used does not coincide with that used in Tello and Winkler, ${ }^{27}$ ie, $\mu>(n-2) \chi_{0} / n$ which is manifestly sharper, it is indeed consistent with the previous result. This difference is not surprising and justified by the fact that the technique herein used is not a direct adaptation of that used in Tello and Winkler $^{27}$ (and also in Cao and Zheng ${ }^{28}$ ). More exactly, unlike the linear case corresponding to $m=1$ (and $\alpha=1$ ) in problem (1), the nonlinearity for the diffusion does not allow us to express $u$ through an explicit representation formula; in particular, its boundedness properties are not promptly addressed by the Neumann heat semigroup theory, so that it is necessary to use Lemma A.1. of Tao and Winkler ${ }^{35}$ to investigate them. 


\section{5 | NUMERICAL SIMULATIONS}

In this section, we test the presented theoretical results by numerically simulating system (1) in 1, 2, and 3 dimensions. Specifically, we investigate whether the solutions are globally bounded, or whether they blow-up in finite time.

The solution algorithm is based on an adaptive, implicit Runge-Kutta finite element method. ${ }^{36}$ Since we are looking for regions where solutions are unstable, if such a solution is found, the discretisation must be increased to ensure that this outcome is the true numerical solution, rather than a numerical artefact. Here, whenever a solution was observed to be unstable, the grid was refined to have ten times as many elements as previously simulated, to ensure the outcome. Critically, the complexity of the simulation grows rapidly as the dimension of the domain of the problem is increased to 2 or 3. Specifically, in higher spatial dimensions, larger numbers of finite elements are required to provide the same level of discretisation as simulations in lower spatial dimensions. However, increasing grid refinement dramatically increases the scale of the problem and slows down the solution production speed. Alternatively, we can shrink the spatial scale of the domain over which are solving. This allows us to refine smaller and smaller pieces of Euclidean space, without increasing the grid refinement. However, by shrinking the solution domain, we may miss out interesting spatial behaviour, since it is well known that the domain size is often a critical bifurcation parameter for the production of heterogeneous solutions. ${ }^{37-39}$ Thus, if a solution is presented as tending to a spatially homogeneous state this is only true for the domain size presented in the figure. Simulating the solution on larger domains would require much higher spatial refinement than our current computing abilities can provide.

We, first, investigate the influence of $m$ and $\alpha$ on the solution, in accordance to the ranges chosen in this investigation. Critically, $m$ and $\alpha$ control the diffusion and cross diffusion components, respectively, in system (1). Figure 1A presents a simulated parameter sweep over multiple values of $m \in \mathbb{R}$ and $\alpha \leq 1$. We immediately observe that parameter $m$ has a
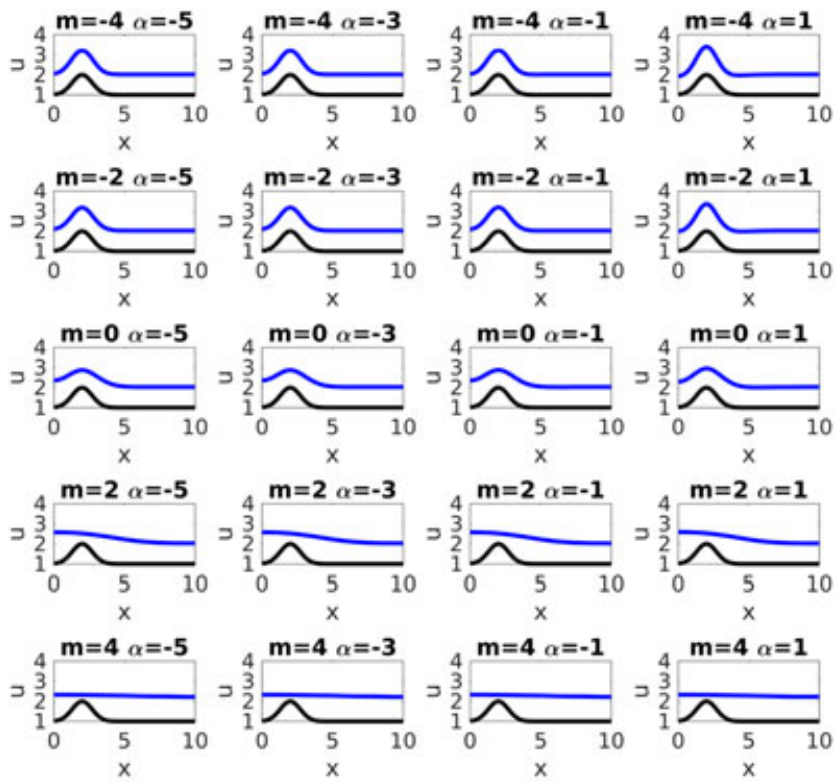

(A)
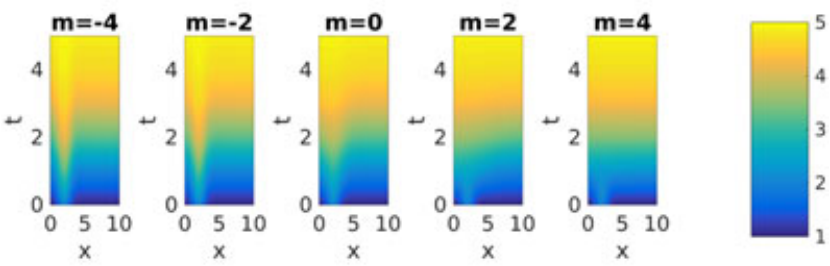

(B)

FIGURE 1 Simulating system (1) in one dimension. Parameters are $a=1, b=2, \chi_{0}=1, k=1$, and $\mu=0.2$. Other parameters noted on the figures. The initial condition is $1+\exp \left(-(x-2)^{2}\right)$ in each case. In (A) we consider the effect of varying parameters $m$ and $\alpha$. The black line is the initial condition and the blue line is the solution at $t=1$. In (B) we fix the value of $\alpha=-0.1$ and vary $m$. Using a space-time plot, we demonstrate that the simulation tends to the homogeneous steady state, as defined by the logistic contribution to system (1). The colour bar on the right specifies the size of $u$ [Colour figure can be viewed at wileyonlinelibrary.com] 


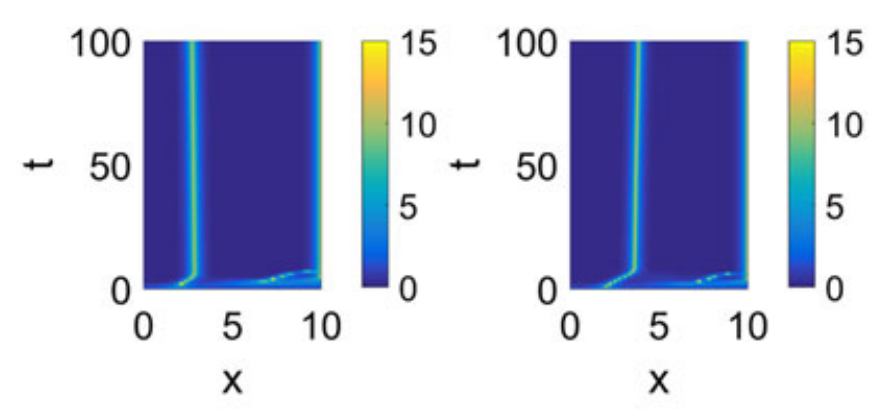

(A) $\alpha=0.9$

(B) $\alpha=1.1$
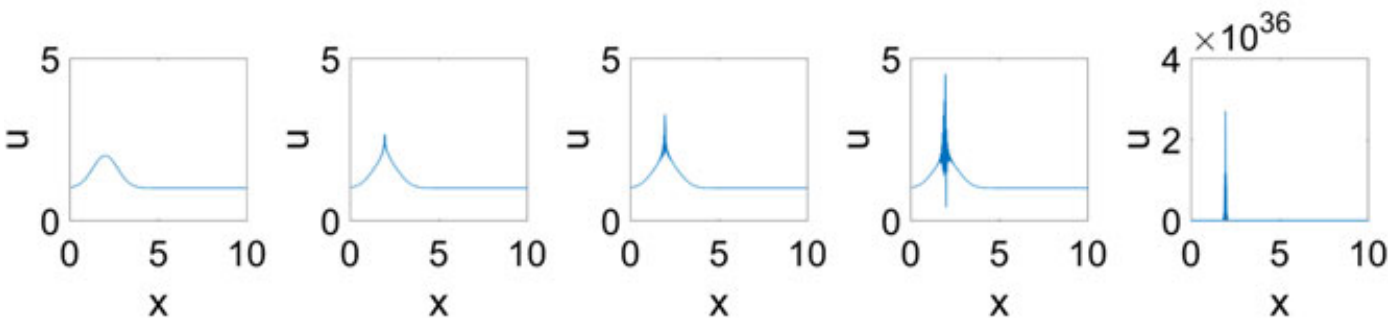

(C) $\alpha=10$

FIGURE 2 Simulating system (1) in one dimension with different values of $\alpha$. Parameters are $b=0, \chi_{0}=1, k=1, m=0$ and $\mu=0.2$, with $\alpha$ specified beneath each figure. The initial condition is $1+\exp \left(-(x-2)^{2}\right)$ in each case. In (A) and (B) the simulations tend to a bounded, heterogeneous concentrations of $u$. The colour bar of $u$ is given on the right of each (A) and (B). If $\alpha$ is increased too far, as in (C), we observe that $u$ becomes spatially unstable and grows without bound as time increases. the simulation time increases left to right and the plots are illustrated at times $t=0,7.3 \times 10^{-6}, 8.3 \times 10^{-6}, 9 \times 10^{-6}$, and $9.0714 \times 10^{-6}$ [Colour figure can be viewed at wileyonlinelibrary.com]

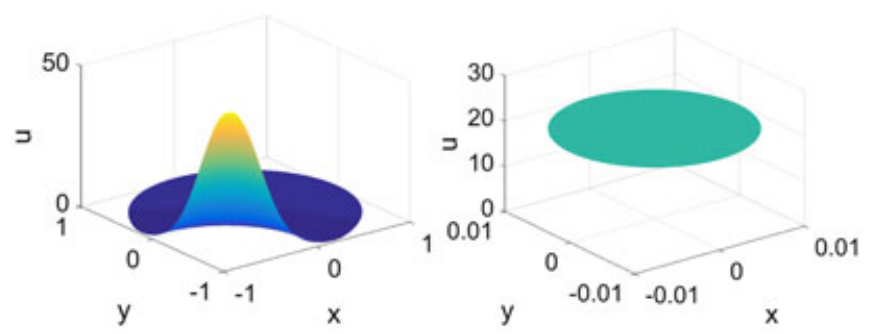

(A) $\alpha=0.1$

(B) $\alpha=0.6$
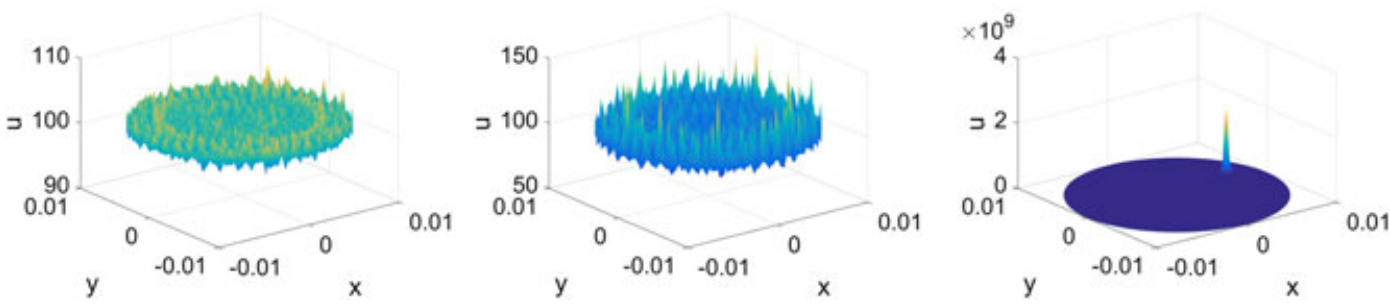

(C) $\alpha=3$

FIGURE 3 Simulating system (1) on a 2-dimensional disk, with different values of $\alpha$. Parameters are $b=0, \chi_{0}=2, k=1, m=0.1$, and $\mu=0.05$. The initial condition is $100\left(1+\exp \left(-x^{2}-y^{2}\right)\right)$ in each case. The values of $\alpha$ are denoted beneath each subfigure. (A) and (B) illustrate that if $\alpha$ is small enough then the simulation is bounded for all time, even for a very weak dampening coefficient $\mu$. The simulations in (A) and (B) have been run for 100 time units and have reached their, respective, stationary states. As $\alpha$ is increased, we see in (C) that the simulations begin to demonstrate the same instability seen in Figure $2 \mathrm{C}$, namely, as the time increases (plots are illustrated at $t=2.2 \times 10^{-5}$, $2.3 \times 10^{-5}$ and $2.3214 \times 10^{-5}$, left to right, respectively) $u$ becomes spatially unstable and grows without bound [Colour figure can be viewed at wileyonlinelibrary.com] 
bigger influence on the simulation than $\alpha$. Namely, as $m$ decreases it takes longer for spatial heterogeneities to disappear. This, of course, makes sense as the diffusion rate is $(u+1)^{m-1}$ and, thus, for $m<1$ the diffusion rate is smaller at local maxima of $u$.

Having discovered the insensitivity to $\alpha$, we present space-time simulations of $u$, varying just $m$, in Figure 1B.Here, we are able to visualise the removal of spatial heterogeneities over time. Combining the insights from Figure 1A,B, we observe that in general the population size tends to be dominated by the logistic kinetics (compare the black and blue lines of Figure 1A), whereas it is the time to homogeneity that is controlled by the diffusive and cross diffusion parameters. Specifically, we see that, even in the case slow diffusion $(m<1)$, there is a rapid global convergence from the initial condition to the spatially homogeneous solution of the logistic kinetics, $k / \mu=5$, whereas local heterogeneities take longer to smooth out (see Figure 1B).

In the case of one dimension, $n=1$, the theory presented here suggests that for $m \in \mathbb{R}$, the simulations converge for all $\alpha<1$ regardless the size of $\mu$. In Figure 2A, we see that this holds true as $u$ tends to a stationary, spatially heterogeneous, bounded solution. Moreover, Figure 2B demonstrates that the inequality is not tight because $u$ still converges to a stationary, spatially heterogeneous, bounded solution even though the inequality is violated. However, if $\alpha$ is increased too far (Figure 2C) we see that the population becomes unstable. Namely, the peak of the initial heterogeneity becomes sharper and high frequency spatial oscillations begin to appear, rapidly spreading out from this localised peak. Eventually, the breakdown in convergence leads to a solution blowing up to a value over $10^{36}$.

When we simulate system (1) in 2 dimensions, we see a similar trend when increasing $\alpha$. Namely, when $\alpha<1$, $u$ remains bounded for all time and converges to a stationary state. Moreover, if the 2-dimensional space is large enough then spatial heterogeneity can be supported (compare Figure 3A,B). However, if $\alpha$ is increased too far (see Figure 3C), we observe that high frequency oscillations begin to rapidly grow (compare with Figure $2 \mathrm{C}$ ) and the solution grows to over $10^{9}$ in less than $10^{-4}$ time units.

Next, we simulate system (1) in 3 dimensions, for $b=0$ and $m \geq 1$. We note that in this case, the theory establishes that when $\alpha+1<\max \{2, m+2 / n\}=\max \{2, m+2 / 3\}$, the solutions are all bounded, regardless the size of $\mu$. Once again, we see that the boundedness of $u$ depends critically on $\alpha$. However, the previously mentioned bound is not optimal. Specifically, as seen in Figure 4A, the population of $u$ is bounded even though the inequality is violated. Although not explicitly shown, the $\alpha=10$ case rapidly converges to the homogeneous steady state defined by the logistic kinetics, $k / \mu=20$.

Critically, the dynamics of the unbounded $u$ solutions in 3 dimensions seem to be subtler than those seen in Figures 2 and 3. Specifically, although for large $\alpha$ the solution $u$ does grow without bound exponentially fast (see Figure 4C), the solution does not rapidly explode when $\alpha=20$. Instead, as is seen in Figure 4B, the maximum of $u$ grows linearly over time.

Finally, we simulate the effect of varying $b$ on the solution in 3 dimensions. Here, we assume that $\alpha$ is smaller than 1 to ensure that the solution is always bounded, for any $m \in \mathbb{R}$ and regardless $\mu$. When $b=0$, we are able to support stable, stationary, heterogeneous solutions of $u$ (see Figure 5A). Whereas when $0<b \leq 2$ the solution rapidly homogenises and tends to the stable stationary state defined by the logistic kinetics, $k / \mu=20$.

In summary, these simulations illustrate the veracity of the results contained within this paper. Specifically, global boundedness of system (1) depends on the spatial dimension we are considering, as well as the diffusive and cross-diffusive parameters of the system.

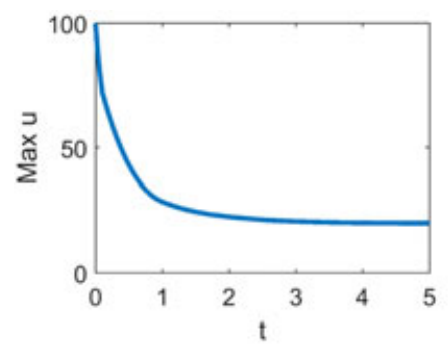

(A) $\alpha=10$

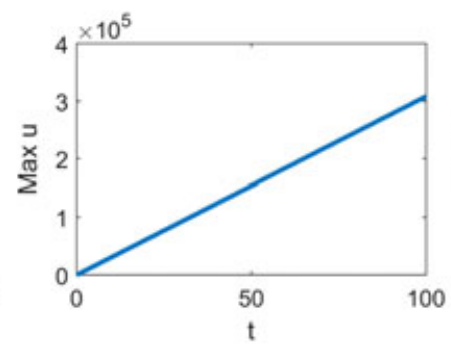

(B) $\alpha=20$

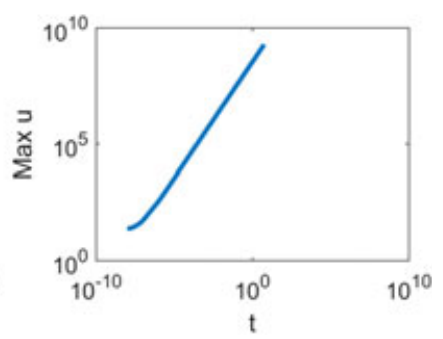

(C) $\alpha=30$

FIGURE 4 Simulating system (1) within a 3-dimensional sphere, with different values of $\alpha$. Here, we only plot the maximum value of $u$ throughout the simulation. Parameters are $b=0, \chi_{0}=2, k=1, m=1.5$ and $\mu=0.05$. The values of $\alpha$ are denoted beneath each subfigure. Critically, in (A), the simulation is bounded for all time. However, as $\alpha$ is increased $\max (u)$ appears to grow without bound. Specifically, the growth is linear in time in (B) and exponentially fast (C) (note the logarithmic axes) [Colour figure can be viewed at wileyonlinelibrary.com] 


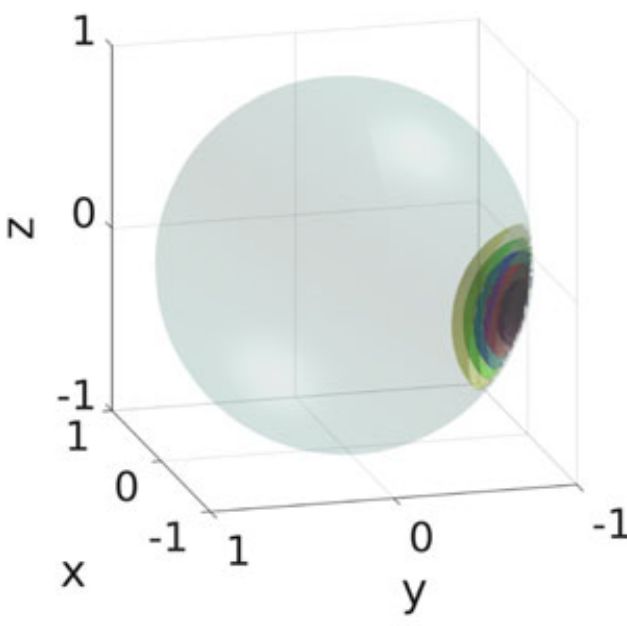

(A)

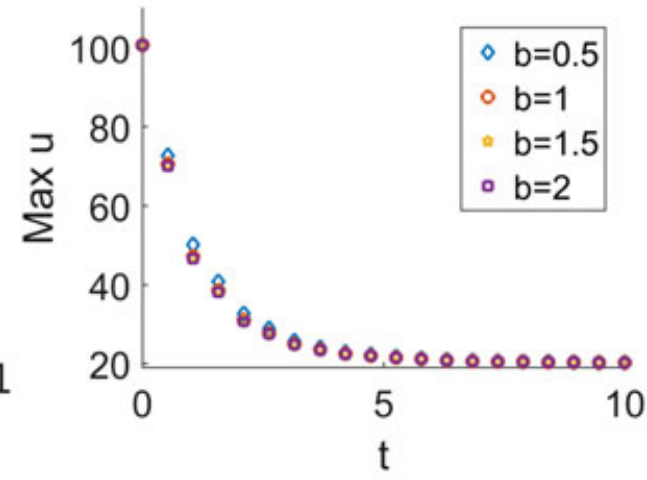

(B)

FIGURE 5 Illustrating the influence of $b$. Parameters are $a=1 \alpha=0.1, \chi_{0}=2, k=1, m=0.1$ and $\mu=0.05$. The value for $b$ is 0 in (A) and noted in the legend of (B). The initial condition is $100\left(1+\exp \left(-x^{2}-y^{2}-z^{2}\right)\right)$ in each case. (A) illustrates that $u$ can support bounded and stationary heterogeneous concentrations when $b=0$. Here, we are visualising the isosurfaces $u=20$ (yellow), 30 (green), 40 (blue), 50 (red), and 60 (black) inside the sphere of unit radius (grey). The simulation was run for 100 time units and did not significantly change for the last 50 time units. In (B), we illustrate the influence of increasing $b$ on the value of $\max (u)$. In each case, we see that the simulation is always bounded [Colour figure can be viewed at wileyonlinelibrary.com]

\section{ACKNOWLEDGEMENTS}

The authors are grateful to the referees for helpful suggestions which improved this article. GV is a member of the Gruppo Nazionale per l'Analisi Matematica, la Probabilità e le loro Applicazioni (GNAMPA) of the Istituto Nazionale di Alta Matematica (INdAM) and gratefully acknowledges the Italian Ministry of Education, University and Research (MIUR) for the financial support of Scientific Project "Smart Cities and Communities and Social Innovation-ILEARNTV anywhere, anytime-SCN_00307.”

\section{ORCID}

\section{Giuseppe Viglialoro (D) http://orcid.org/0000-0002-2994-4123}

\section{REFERENCES}

1. Belmonte-Beitia J, Woolley TE, Scott JG, Maini PK, Gaffney EA. Modelling biological invasions: individual to population scales at interfaces. J Theor Biol. 2013;334(0):1-12.

2. Keller EF, Segel LA. Initiation of slime mold aggregation viewed as an instability. J Theor Biol. 1970;26(3):399-415.

3. Horstmann D. From 1970 until present: the Keller-Segel model in chemotaxis and its consequences I. Jahresberichte DMV. 2003;105(3):103-165.

4. Hillen T, Painter KJ. A user's guide to PDE models for chemotaxis. J Math Biol. 2009;58(1):183-217.

5. Osaki K, Yagi A. Finite dimensional attractor for one-dimensional Keller-Segel equations. Funkcial Ekvacioj. 2001;44(3):441-470.

6. Horstmann D, Wang G. Blow-up in a chemotaxis model without symmetry assumptions. Eur J Appl Math. 2001;12(2):159-177.

7. Winkler M. Finite-time blow-up in the higher-dimensional parabolic-parabolic Keller-Segel system. J Math Pures Appl. 2013;100(5):748-767.

8. Payne LE, Song JC. Lower bounds for blow-up in a model of chemotaxis. J Math Anal Appl. 2012;385(2):672-676.

9. Jäger W, Luckhaus S. On explosions of solutions to a system of partial differential equations modelling chemotaxis. T Am Math Soc. 1992;329(2):819-824.

10. Nagai T. Blowup of nonradial solutions to parabolic-elliptic systems modeling chemotaxis in two-dimensional domains. $J$ Inequal Appl. 2001;6(1):37-55

11. Cieślak T, Morales-Rodrigo C. Quasilinear non-uniformly parabolic-elliptic system modelling chemotaxis with volume filling effect. existence and uniqueness of global-in-time solutions. Topol Methods Nonlinear Anal. 2007;29(2):361-381.

12. Cieślak T, Winkler M. Finite-time blow-up in a quasilinear system of chemotaxis. Nonlinearity. 2008;21(5):1057-1076.

13. Horstmann D, Winkler M. Boundedness vs. blow-up in a chemotaxis system. J. Differerential Equations. 2005;215(1):52-107. 
14. Marras M, Vernier-Piro S, Viglialoro G. Blow-up phenomena in chemotaxis systems with a source term. Math Method Appl Sci. 2016;39(11):2787-2798.

15. Winkler M. Does a 'volume-filling effect' always prevent chemotactic collapse?Math Method Appl Sci. 2010;33(1):12-24.

16. Winkler M, Djie KC. Boundedness and finite-time collapse in a chemotaxis system with volume-filling effect. Nonlinear Anal Theory Methods Appl. 2010;72(2):1044-1064.

17. Verhulst P-F. Notice sur la loi que la population poursuit dans son accroissement. Correspondance Mathématique et Physique. $1838 ; 10: 113-121$.

18. Funaki M, Mimura M, Tsujikawa T. Travelling front solutions arising in the chemotaxis-growth model. Interface Free Bound. 2006;8:223-245.

19. Funaki M, Tsujikawa T. Aggregating pattern dynamics in a chemotaxis model including growth. Physica A. 1996;230(3-4):499-543.

20. Marras M, Viglialoro G. Blow-up time of a general Keller-Segel system with source and damping terms. $C R$ Acad Bulg Sci. 2016;69(6):687-696.

21. Viglialoro G. Blow-up time of a Keller-Segel-type system with Neumann and Robin boundary conditions. Diff Int Eqns. 2016;29(3-4):359-376.

22. Lankeit J. Eventual smoothness and asymptotics in a three-dimensional chemotaxis system with logistic source. J Differerential Equations. 2015;258(4):1158-1191.

23. Winkler M. Boundedness in the higher-dimensional parabolic-parabolic chemotaxis system with logistic source. Commun Part Diff Eq. 2010;35(8):1516-1537.

24. Viglialoro G. Very weak global solutions to a parabolic-parabolic chemotaxis-system with logistic source. J Math Anal Appl. 2016;439(1):197-212.

25. Viglialoro G. Boundedness properties of very weak solutions to a fully parabolic chemotaxis-system with logistic source. Nonlinear Anal Real World Appl. 2017;34:520-535.

26. Viglialoro G, Woolley TE. Eventual smoothness and asymptotic behaviour of solutions to a chemotaxis system perturbed by a logistic growth. Discrete Continuous Dyn Syst Ser B. 2017. https://doi.org/10.3934/dcdsb.2017199

27. Tello JI, Winkler M. A chemotaxis system with logistic source. Commun Part Diff Eq. 2007;32(6):849-877.

28. Cao X, Zheng S. Boundedness of solutions to a quasilinear parabolic-elliptic Keller-Segel system with logistic source. Math Meth Appl Sci. 2014;37(15):2326-2330.

29. Zheng J. Boundedness of solutions to a quasilinear parabolic-elliptic Keller-Segel system with logistic source. J Differential Equations. 2015;259(1):120-140.

30. Wang $\mathrm{L}, \mathrm{Mu} \mathrm{C}$, Zheng $\mathrm{P}$. On a quasilinear parabolic-elliptic chemotaxis system with logistic source. J Differerential Equations. 2014;256(5):1847-1872.

31. Xie L, Zhaoyin X. Boundedness in quasilinear Keller-Segel equations with nonlinear sensitivity and logistic source. Discrete Contin Dyn Syst Ser A. 2015;35(8):3503-3531.

32. Cieślak T. Quasilinear nonuniformly parabolic system modelling chemotaxis. J Math Anal Appl. 2007;326:1410-1426.

33. Gilbarg D, Trudinger N. Elliptic Partial Differential Equations of Second Order. 2nd ed. Berlin: Springer-Verlag; 1983.

34. Ladyženskaja OA, Solonnikov VA, Ural'ceva NN. Linear and quasi-linear equations of parabolic type. Translations of Mathematical Monographs, Vol. 23. Providence: American Mathematical Society; 1988.

35. Tao Y, Winkler M. Boundedness in a quasilinear parabolic-parabolic Keller-Segel system with subcritical sensitivity. $J$ Differerential Equations. 2012;252(1):692-715.

36. Ascher UM, Ruuth SJ, Spiteri RJ. Implicit-explicit Runge-Kutta methods for time-dependent partial differential equations. Appl Numer Math. 1997;25(2-3):151-167.

37. Maini PK, Woolley TE, Baker RE, Gaffney EA, Lee SS. Turing's model for biological pattern formation and the robustness problem. Interface Focus. 2012;2(4):487-496.

38. Woolley TE, Baker RE, Maini PK, Aragón JL, Barrio RA. Analysis of stationary droplets in a generic Turing reaction-diffusion system. Phys Rev E. 2010;82(5):051929-1-051929-9.

39. Woolley TE, Baker RE, Tickle C, Maini PK, Towers M. Mathematical modelling of digit specification by a sonic hedgehog gradient. Dev Dynam. 2014;243(2):290-298.

How to cite this article: Viglialoro G, Woolley TE. Boundedness in a parabolic-elliptic chemotaxis system with nonlinear diffusion and sensitivity and logistic source. Math Meth Appl Sci. 2018;41:1809-1824. https://doi.org/10.1002/mma.4707 